\title{
Think Indifferent: On the Perils Of Scientific Deliberation, Without Attention For Critical Evaluation
}

\author{
Fred Hasselman ${ }^{1,2^{*}}$, Sander V. Crielaard ${ }^{1}$ and Anna M. T. Bosman ${ }^{1,2}$ \\ ${ }^{1}$ School of Pedagogical and Educational Science, Radboud University Nijmegen \\ ${ }^{2}$ Behavioural Science Institute, Radboud University Nijmegen
}

*Corresponding Author:

Fred Hasselman

School of Pedagogical and Educational Science

Montessorilaan 3

P.O. Box 9104

6500 HE Nijmegen

The Netherlands

f.hasselman@pwo.ru.nl

Key-words:

Theory evaluation, expert peer-review; post-publication peer-review; Marginal results Evaluation Quality; Bones' $\Psi$; Simplest Tests of Hypotheses Possible; predictive power; empirical accuracy; unconscious thought theory.

Word count: 4978 [max. 5000] (includes paragraph headings, footnotes, epigraph)

Abstract word count: 142 [max. 150]

Reference count: $50 \quad$ [max. 50] 
Think Indifferent:

On the Perils Of Scientific Deliberation, Without Attention For Critical Evaluation

\begin{abstract}
Replication attempts of empirical psychological phenomena lack guidance by a larger effort to evaluate the psychological theory that predicted the phenomena. In this paper we present a template for theory evaluation suited for the class of theories produced by psychological science: Theories of construction. As a case study, we perform a rigorous post-publication peer-review of the theoretical core of Unconscious Thought Theory (UTT). We present several uncomplicated indices that quantify the quality of evaluation of the results and conclusions by experts who reviewed the article and the amount of interpretation bias on behalf of the authors. The results reveal a failure of expert peer-review to detect empirical reports of sub-standard quality. The analyses reveal there is in fact hardly any empirical evidence for the predictions of UTT in important papers that claim its support. Our advice is to evaluate before you replicate.
\end{abstract}

"If Psychology is ever to become anything more than a mere aggregation of opinions, it can only be by the establishment of some datum universally agreed to."

- Herbert Spencer (1855, p. 8)

\title{
To make a difference...
}

Did you hear the one about the difference between the current crisis of confidence in psychology as an empirical science (cf. Pashler \& Wagenmakers, 2012), the one in the 1990s (cf. Hogg \& Grieve, 1999), the one in the 1980s (cf. Wong, 1981) and the one in the 1970s (cf. Elms, 1975)? Indeed, there is no difference! A recent article estimated that in the last 100 years, $0.1 \%$ of studies in psychological science concerned exact replication attempts of empirical findings (Makel, Plucker, \& Hegarty, 2012). This suggests Spencer's datum in the epigraph is as remotely removed from us now, as it was 158 years ago, justifying the necessity of recent proposals to improve on this statistic (e.g. Asendorpf et al., 2013; Open Science Collaboration, 2012).

In this paper we argue that, to finally make a difference, a "daisy-chain" of replications of a single empirical phenomenon will not suffice (e.g., Bosman, Cox, Hasselman, \& Wijnants, in press). Psychological science may have advanced beyond an aggregation of opinions, it is not a discipline of science known for producing theories about reality that make increasingly accurate predictions by correcting invalid theoretical claims (e.g. Ioannidis, 2012). Such observations show both the necessity and difficulty of formal theory evaluation in psychological science: When does a failed exact replication of an empirical phenomenon damage the scientific credibility of the theoretical whole that predicted the phenomenon? We propose a template procedure for the evaluation of predictions by psychological theories that involves a rigorous post-publication peer-review of empirical reports that claim support for the theory under scrutiny. The aim is to assess the credibility of the core hypotheses of the theory, which eventually should guide exact replication attempts. As a case study we evaluate the theoretical core of Unconscious Thought Theory (UTT, described in the Methods section). Before we present our case, we discuss our perspective on theory evaluation in psychological science.

\section{Can theories of psychology be evaluated for their scientific credibility?}

We suggest the crises of confidence about the empirical record periodically emerge due to an unresolved foundations debate. Throughout its history as an empirical science, psychology has been criticized for pretending it studies a physical system like a natural science does, when in fact: "[...] 
psychology as a science, devoid of all postulating of "deeper-lying entities," does nothing of the kind. It assumes only the phenomena - the thoughts and feelings as actually known, and the possibility of ascertaining uniform relations among them." (Ladd, 1892, pp. 29-30, emphasis added, reviewing William James' "The Principles of Psychology"). Some would call these theories phenomenological, but we prefer Einstein's terminology (see van Dongen, 2010) ${ }^{1}$ and use theories of construction as a contrast to theories of principles that are mainly produced by the natural sciences (see Figure 1).

Constructed theories consist of chains of conceptual replications of interesting patterns in the empirical record, originating from a theoretical core (cf. Lakatos, 1976). The core is a regular part of the empirical record containing phenomena that were constructed to constitute a consistent theoretical whole. The core is often protected by a boundary of auxiliary hypotheses that can easily be adjusted, or sacrificed in order to 'save' the phenomena that constitute it (Lakatos, 1976). The latter is the most important difference between theories of construction and theories of principles. Principled theories are "closed" (cf. Bokulich, 2004), they break down as a whole when a crucial experiment does not yield results predicted by the theory: "[...] if an experiment does not fit in Newtonian physics, you don't know what you mean by the words." (Heisenberg interviewed by Kuhn, 1963, p. 24, February 27th). These theories allow for a program of strong inference (e.g. Platt, 1964): A single datum can be obtained in a crucial experiment to decide between the veracity of competing theoretical claims.

This raises concerns about whether theory evaluation in psychological science can be achieved in the spirit of the scientific method of strong inference developed to evaluate predictions by theories of principles (e.g. Fiedler, Kutzner, \& Krueger, 2012). There is, however, more to formal scientific theory evaluation than concocting a crucial measurement context. Theories of principles accumulate a "trackrecord" of the following properties: Predictive power (the extent to which theories are able to predict a measurement context in which a phenomenon may be observed) and empirical accuracy (the difference between predicted and observed measurement outcomes). We examine whether these properties can be used to evaluate theories of construction as well.

Suppose the number of positive results published in a discipline of science is indicative of the predictive power of the theories proposed by the discipline. Then psychology produces theories with uncanny predictive powers: $91.5 \%$ of examined publications contained positive findings (Fanelli, $2010)^{2}$. If these theories compete to explain the same phenomena, the ones that most accurately predict measurement outcomes should be evaluated as theories with the highest scientific credibility. Apparently psychological science currently fails to do so, as the number of competing theories is growing rather than shrinking and this is a sign of an unhealthy state of theory evaluation and revision (see for a thorough health check Hasselman, Cox \& Seevinck, submitted).

\footnotetext{
${ }^{1}$ We thank Michael Seevinck for introducing us to this important history of science.

${ }^{2}$ Former supermodel and part-time philanthropist Arina K. Bones (2012) used these findings to reveal a failure in the peerreview of a series of studies by Daryl Bem in which he showed pictures of naked people to students. Based on the interpretation of his results as the evolved ability of male participants to perceive the occurrence of future erotic events, evidence for the existence of Psi was inferred. Bones proved Psi had already been inferred from the uncanny ability of scientists to correctly predict outcomes of their experiments.
} 


\section{Theory of construction empirical science?}

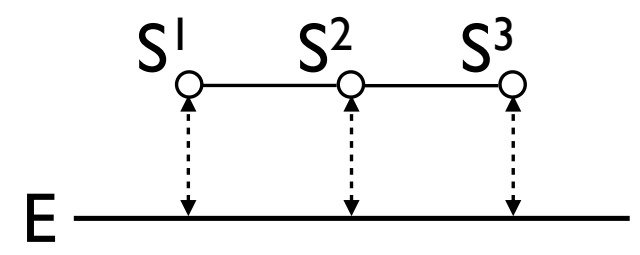

- "devoid of all postulating of 'deeper-lying entities' " There is no (explicit) formal system of axioms and postulates. A theory (S1-3) is constructed by association or conceptual replication. There is no consensus in the scientific community about the system it studies, what the interesting phenomena and levels of analysis are.

- "It assumes only the phenomena" Phenomena are induced directly from the manifold of immediate sensory experiences $\mathbf{E}$, as empirical laws $\mathbf{S}$.

- "possibility of ascertaining uniform relations among them" Predictions by a core of $\mathbf{S}$ concern the observation of correlated phenomena at some level of confidence. $\mathbf{E}$ informs and adjusts $\mathbf{S}$ by induction.

- Theory Evaluation

Step I: Identify the theoretical core (e.g. S1, S2, S3)

Step II: Identify the core hypotheses

Step III: Scrutinize the empirical evidence

Step IV: Estimate predictive power and empirical accuracy

\section{Theory of principles consensus formalism science}

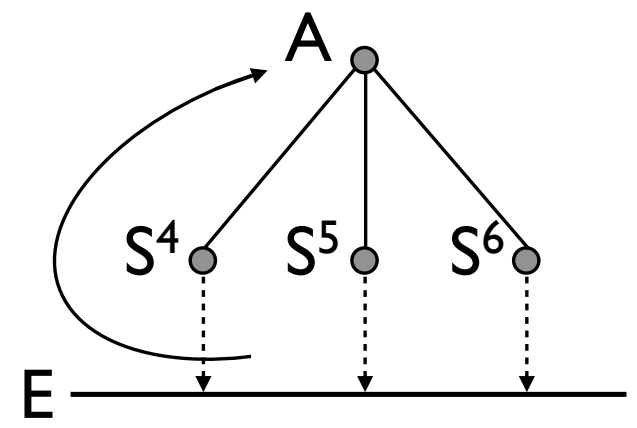

- Consensus Formalism

A formal system of axioms $\mathbf{A}$, is raised from conjecture to postulate (the upward arrow). A describes a specific domain in reality for a discipline of science to study; a system, its relevant phenomena and levels of analysis. There is a consensus in the scientific community about the truth status of the formalism.

- Closed Theory

Laws $\mathbf{S}$ are deduced from the formalism and a theory (S4-6) competes for scientific credibility within the domain described by A. There is no logical route from $\mathbf{E}$ to $\mathbf{A}$, only from $\mathbf{A}$ to $\mathbf{S}$ to $\mathbf{E}$.

- Accurate Prediction

A theory predicts a measurement context in $\mathbf{E}$ in which phenomena may be observed in terms of measurement outcomes. $\mathbf{E}$ cannot adjust $\mathbf{S}$, a prediction is accurate or not.

- Theory Evaluation

Competing predictions are tested under conditions of strong inference; predictive power and empirical accuracy are assessed over a number of such tests.

Figure 1. An adaptation of the "Solovine Schema" drawn by Albert Einstein in 1954 in a letter to his friend Maurice Solovine (see van Dongen 2010, p. 52-53).

How to establish the empirical accuracy of a theory of construction? There are several options. A recent study used the meta-analysis summary effect size $(E S)$ to evaluate whether studies that composed an analysis were sufficiently powered to detect the magnitude of the summary ES (Button et al., 2013). Severely underpowered studies were observed (between $\sim 8 \%$ and $\sim 31 \%$ ) and in terms of empirical accuracy, this means that the theory did not predict measurement outcomes beyond the magnitude of the significance threshold needed to confirm the observation of a phenomenon with some confidence. The predictive power of psychological theories is high, but their empirical accuracy is low. It is beyond the scope of this paper to discuss, but we consider Effect Size Confidence-Interval hypothesis testing (ES-CI), as a more sophisticated way to estimate the empirical accuracy of predictions (Cumming, 2012).

Our perspective is similar to the "Deepness of hypotheses tested"-explanation suggested for the positive publication bias in psychological science: A young science is mainly concerned with the observation of phenomena with some level of confidence (shallow hypotheses), whereas a mature science tests hypotheses that "[...] reach deeper levels of reality, are logically stronger, less likely to be true, and are more conclusively testable [...]" (Fanelli, 2010, p. 6). This reflects the distinction between "constructive" and "principled" theories, but also the weakness of the knowledge system in empirical psychology (LeBel \& Peters, 2011). 


\section{The present study: Something to work with}

Given our assessment of the nature of theories in psychological science, we suggest that replication attempts, whether conceptual or exact, should be targeted, or orchestrated by an evaluation of the scientific credibility of the theoretical core that predicted the original observation. A successful exact replication by itself provides support for a specific empirical phenomenon and not necessarily to a larger theoretical whole. A failed targeted replication of the core phenomena will be more informative than refuting an auxiliary hypothesis, which has little consequence for the scientific credibility of the core itself. Evidently, if a replication attempt fails for a prediction by a theory that could have been evaluated as trivial by a post-publication peer-review, the resources spent on replication could have been put to better use.

How to proceed? Applying the tools that were developed to evaluate theories of principles will not be possible until psychological science starts to produce such theories and right now we need something to work with. Our proposal is to evaluate the theoretical core of a theory of construction, thereby avoiding confirmed, rejected or failed-to-replicate predictions by auxiliary hypotheses that in the end have no say whatsoever at the level of the constructed whole. Auxiliary hypotheses are easily identified; they concern the elegant experimental condition that was attempted only once, the correlated dependent variable, or the impressive stimuli that quietly left the symposium, never to be heard of again. The core hypotheses on the other hand will be reported in high-impact publications that claim empirical confirmation of just a few phenomena, explicitly predicted by the theory. The theory itself is actually a construct of those empirical phenomena (see Figure 1), it most certainly cannot be considered derived from a set of postulates or axioms about the structure of a specific domain in reality (a formalism is however always implicitly present in scientific theorizing, e.g. Molenaar, 2008).

If the core does not collapse during the evaluation procedure we suggest the theory should gain a substantial amount of scientific credibility in terms of predictive power. When the results indicate a breach of the core is imminent, we expect this will be due to logical inconsistencies, contradicting predictions and probably most common, the inability to generate consistent empirical support across all examined studies, which may be evidenced by (public) displays of p-hacking or the use of other researcher degrees of freedom (John, Loewenstein, \& Prelec, 2012; Simmons, Nelson, \& Simonsohn, 2011).

The primary goal of our evaluation is to provide a template procedure, open to adjustment and refinement, which can be used to evaluate any theory of psychological science of which a constructed theoretical core can reasonably be identified. A secondary goal is to assess whether theory evaluation is actually achieved by the expert peer-review procedure, by quantifying issues with analysis strategies, reporting of results and validity of inferred scientific knowledge that can be found in published articles. We suggest several uncomplicated indices that aid in the evaluation of the quality of the peer-review procedure and the predictive power of the core.

\section{Method}

\section{Step I: Identifying the core of Unconscious Thought Theory (UTT)}

The theory whose core we will evaluate is Unconscious Thought Theory ${ }^{3}$ (Dijksterhuis \& Nordgren, 2006). According to UTT, the best strategy to pursue when faced with a complex problem is to leave its resolution to the superior skills of the unconscious mind. The empirical phenomenon has been called the "deliberation-without-attention effect" or the Unconscious Thought Effect (UTE), but conceptually, this particular phenomenon is known to science as "unconscious cerebration or

\footnotetext{
${ }^{3}$ The decision to evaluate UTT was not random, but can be considered trivial. Our initial involvement with the subject was due to an undergraduate project (academic year 2010-2011) that attempted a conceptual replication of JESP2008 and PsySci2010.
} 
elaboration" (Browne, 1870, p. 235), or incubation - "laying aside a problem as a step toward a solution" (Woodworth \& Schlosberg, 1954, p. 838). Completely separate from UTT, a line of contemporary high-impact incubation research exists (Sio \& Ormerod, 2009 is a meta-analytic review). Associated topics are consolidation in learning (Robertson, 2004) and emergence of insight (insight in problem solving: Stephen, Dixon, \& Isenhower, 2009; creative insight: Stickgold \& Walker, 2004).

Our evaluation concerns four key articles (and an erratum) that together can be described as the theoretical core of UTT. We used several criteria to select these specific publications: 1) The core is published in journals that are the top of their domain: The field of experimental social psychology (Journal of Experimental Social Psychology: JESP), the sub-discipline of social psychology (Journal of Personality and Social Psychology: JPSP), the discipline of psychology (Psychological Science: PsySci) and science in general (Science: SciMag). 2) The theory is well known, but not uncontroversial (e.g., Waroquier, Marchiori, Klein, \& Cleeremans, 2009). Proponents as well as opponents of UTT frequently cite the papers we selected. 3) UTT has many practical applications. Several of the core publications are used to promote the quality of services of commercial endeavors that exploit the predictions by UTT. 4) A meta-analysis is available for the UTE in which the core publications are included. This allows us to get a rough estimate of the empirical accuracy of UTT by evaluating the post-hoc power of the core.

The following publications were used in our evaluation, 1-5 constitute the theoretical core:

1) JPSP2004: Five studies report empirical evidence for the UTE in decision-making. (Dijksterhuis, 2004).

2) SciMag2006: Four studies report the UTE depends on the complexity of the decision problem. We included the online supporting information (Dijksterhuis, Bos, Nordgren, \& van Baaren, 2006a; Dijksterhuis, Bos, Nordgren, \& van Baaren, 2006b)

3) JESP2008: Three studies report on the importance of providing a goal in order to observe the UTE (Bos, Dijksterhuis, \& van Baaren, 2008)

4) PsySci2010: Two studies examine an alternative explanation for UTE and reject it (Strick, Dijksterhuis, \& van Baaren, 2010).

5) PsySci2012: An erratum to PsySci2010 (Strick, Dijksterhuis, \& van Baaren, 2012). The test that rejected the alternative explanation in Study 2 is insignificant.

6) SocCog2011: A meta-analysis of the UTE, providing summary effect sizes and moderator effects (Strick et al., 2011).

\section{Step II: Identifying the core hypotheses and predictions of UTT}

The experimental design most often used to test predictions by UTT is a problem-solving paradigm in which the quality of participants' decisions is compared across the levels of a factor that represents different modes of thought (MOT). There are two core hypotheses that predict a measurement context in which the UTE may be observed: 1) Unconscious thought (UT) is always superior compared to other levels of MOT when making complex decisions. 2) Conscious thought (CT) is inferior to UT when making complex decisions. The first hypothesis is tested as a difference in decision quality between an UT condition, in which participants are distracted from thinking about the problem for a while, and the immediate condition (IM), a baseline in which participants decide immediately after the problem was presented. This is a direct test of the superiority of the UT condition. The second hypothesis predicts a difference in decision quality of the UT condition relative to a condition in which participants were allowed to think about the problem. This is the CT condition and the comparison is a test of the inferiority of CT to UT.

The difference between the predictions is important, because SciMag2006 and JESP2008 do not include an IM condition and those results should be interpreted as tests of an inferior process of conscious thought and not as evidence that supports a superior active unconscious. SciMag2006 states 
that CT decision quality depends on problem complexity, however, for UT: "[...] the quality of choice does not deteriorate with increased complexity, allowing unconscious thought to lead to better choices than conscious thought under complex circumstances, this latter idea being the kernel of the deliberation-without-attention hypothesis." (SciMag2006, p. 1006, emphasis added). This kernel hypothesis translates into explicit predictions of observed cell differences; an effect of problem complexity on decision quality in CT but not for UT and CT should outperform UT under simple circumstances.

The core of UTT is not explicit about differences between CT and IM, though we encountered references to statistical tests comparing the contrast (see the supporting online material, $\mathrm{SOM}^{4}$ ). Details about CT-IM must have been available, because the contrast was evaluated in SocCog2011. No significant summary or moderator effect sizes were found. Therefore the core theoretical claim of UTT about the effect of MOT on the quality of a complex decision must be: UT $>$ CT $\geq$ IM.

Step III: 3PR - Resuscitating confidence in the empirical record?

The main "tool" we used to evaluate UTT was a rigorous post-publication peer-review (3PR) in which we treated the core publications as freshly submitted manuscripts. It is a fact-check of the scientific quality of a published study, of which systematic reviews and meta-analyses generally assume it was performed during the peer-review process. For each individual study in the core a 3-Page Report is drafted (see SOM). Issues were highlighted in text copied from the original that concerned the analysis strategy (AS), reporting of statistical results (SR) and interpretations and conclusions based on the results (IC). Most issues we identified are uncontroversial and concern an undergraduate level knowledge of statistical inference. In addition we used recent publications on p-hacking (Simmons, Nelson, \& Simonsohn, 2011), misreporting of statistics (Bakker \& Wicherts, 2011), controlling for type-I errors (Vul \& Pashler, 2012) and deficiencies in modal research practice (LeBel \& Peters, 2011) to decide which issues to report. There will be issues that can be disputed and these will mostly concern the IC category, the validity of the interpretation of results.

We take the ratio of the total number of issues to the number of test statistics reported in the results section of a publication, as an index that expresses the Marginal results Evaluation Quality of the original peer-review (MrE-Q, aka "the mystery quotient"). Any value of MrE-Q above 1 indicates something must have gone very wrong during the peer-review procedure: Highly implausible results were accepted for publication. At $0.5 \mathrm{MrE}-\mathrm{Q}$ probably concerns issues due to the occasional copy-paste or repeated error. Awards of excellence for authors, reviewers and editors concern publications whose MrE-Q falls below 0.1. This index can also be calculated per category (partial MrE-Q) in which case values will rarely be above 1 .

\footnotetext{
${ }^{4}$ Available at http://fredhasselman.com -> Supplementary Materials
} 
EVALUATE BEFORE YOU REPLICATE

Table 1

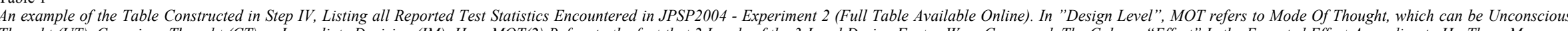

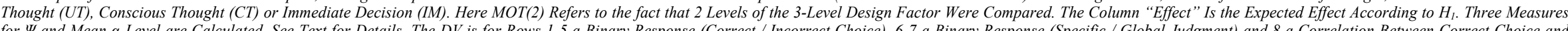

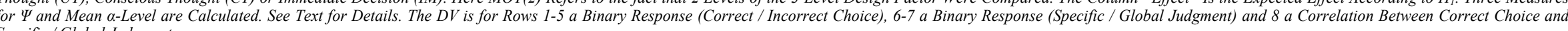

\begin{tabular}{|c|c|c|c|c|c|c|c|c|c|c|c|c|c|c|c|}
\hline \# & Design Level & Effect & As published & $p$ & Effective $\alpha$ & Reject $\mathbf{H}_{0}$ & Recon. Stats & $p$ & Family & Recon. $\alpha$ & Reject $\mathbf{H}_{0}$ & STOHP & $p$ & Adjusted $\alpha$ & Reject $\mathbf{H}_{0}$ \\
\hline 1 & IM & "Better than chance" & $\chi^{2} \mathrm{~s}(92, N=93)>5.19$ & 0.0300 & 0.0500 & YES & $\chi^{2} \mathrm{~s}(1)>5.19$ & ? & $\mathrm{A} 1$ & 0.0167 & - & & & & \\
\hline 2 & СТ & "Better than chance" & $\chi^{2} \mathrm{~s}(92, N=93)>5.19$ & 0.0300 & 0.0500 & YES & $\chi^{2} \mathrm{~s}(1)>5.19$ & $?$ & A2 & 0.0167 & - & & & & \\
\hline 3 & UT & "Better than chance" & $\chi^{2} \mathrm{~s}(92, N=93)>5.19$ & 0.0300 & 0.0500 & YES & $\chi^{2} \mathrm{~s}(1)>5.19$ & ? & A3 & 0.0167 & - & & & & \\
\hline 4 & MOT(2) & $\mathrm{UT}>\mathrm{IM}$ & $\chi^{2}(59, N=60)=3.13^{*}$ & 0.0770 & 0.1000 & YES* & $\chi^{2}(1)=3.13^{*}$ & 0.0770 & B1 & 0.0250 & - & $Z=1.77^{*}$ & 0.0770 & 0.0250 & - \\
\hline 5 & MOT(2) & $\mathrm{UT}>\mathrm{CT}$ & ? & ? & ? & - & $\chi^{2}(1)=0.90^{*}$ & 0.3433 & B2 & 0.0250 & - & $Z=0.95^{*}$ & 0.3430 & 0.0250 & - \\
\hline 6 & MOT(2) & $\mathrm{UT}>\mathrm{IM}$ & ? & $?$ & ? & - & $\chi^{2}(1)=1.03$ & 0.3112 & $\mathrm{C} 1$ & 0.0250 & - & & & & \\
\hline 7 & MOT(2) & $\mathrm{UT}>\mathrm{CT}$ & $\chi^{2}(60, N=61)=6.69$ & 0.0098 & 0.1000 & YES & $\chi^{2}(1)=5.34$ & 0.0209 & $\mathrm{C} 2$ & 0.0250 & YES & & & & \\
\hline \multirow[t]{4}{*}{8} & Sample? & $r>0$ & $r=0.22$ & 0.0400 & 0.0500 & YES & ? & ? & ? & ? & - & & & & \\
\hline & & & \multicolumn{2}{|c|}{ Mean $\alpha$-level: } & 0.0667 & & & & Mean $\alpha$-level: & 0.0214 & & \multicolumn{2}{|c|}{ Mean $\alpha$-level: } & 0.0250 & \\
\hline & & & \multicolumn{2}{|c|}{ All Tests - Bones' $\Psi$} & 1.00 & $6 / 6$ & \multicolumn{3}{|c|}{ All Tests - Reconstructed Bones' $\Psi$} & 0.14 & $1 / 7$ & & & & \\
\hline & & & \multicolumn{2}{|c|}{ Tests of Hypotheses - Bones' $\Psi$} & 1.00 & $1 / 1$ & \multicolumn{3}{|c|}{ Tests of Hypotheses - Reconstructed Bones' $\Psi$} & 0.00 & $0 / 2$ & & 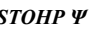 & 0.00 & $0 / 2$ \\
\hline
\end{tabular}

${ }^{*}$ Core Hypotheses 


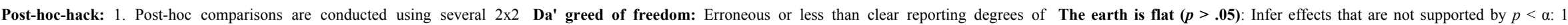
ANOVAs (JPSP2004, SciMag2006, JESP2008,) 2. No adjustments for freedom as evidenced by the test statistic and / or $n$ that are reported for a Based on $p<.08$ or n.s. (JPSP2004, PsySci2012, SocCog2011). 2. Observed $\chi$ multiple comparisons of the $\alpha$-level are reported (JPSP2004, SciMag2006, design cell (JPSP2004, SciMag2006, JESP2008, PsySci2010) p palues were divided by 2 to get a "one-tailed" test (JPSP2004) 3. Most postJESP2008, PsySci2010) 3. Separate Sig. F-tests (1 or 2 main, interaction) reported whereas evaluation is straightforward at the level of the full model hoc effects would be insignificant if $\alpha$ were adjusted for multiple comparison (JPSP2004, SciMag2006, JESP2008,

(JPSP2004, JESP2008, SciMag2006, PsySci2010)

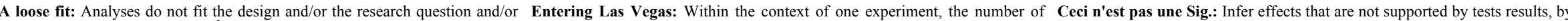
the measured variables: 1. Use $\chi$ as if it were a model with a linear predictor paticipants in design cells appeans to change from analysis to analysis. Theso ensing test results that support a difterent esfect (related to public $p$-hacking): 1 .

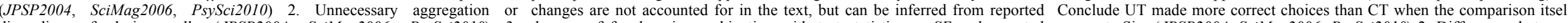

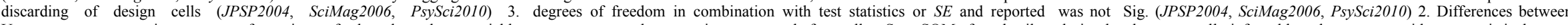

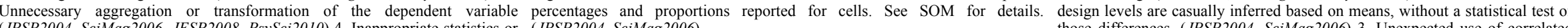
$\begin{array}{ll} & \text { those differences. (JPSP2004, SciMag2006) 3. Unexpected use of correlated }\end{array}$ tests (JPSP2004, SciMag2006, PsySci2010) DVs or factors to suggest a core effect was at work (JPSP2004, SciMag2006, PsySci2010). 4. In JESP2008 the predicted effect is Sig. only for one stimulus set. The prediction is still reported as confirmed, consequences for previous
studies were not discussed.

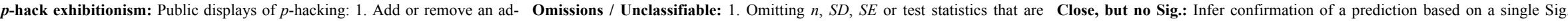

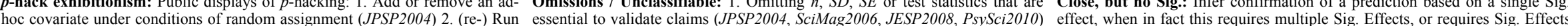

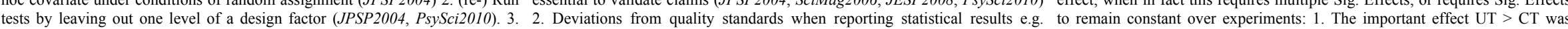

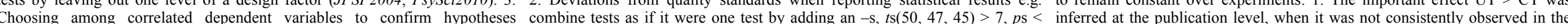
(JPSP2004, PsySci2010) 4. Post-data collection exclusion rule used to discard a .02 ; unknown format for (wrong) $d f$ of $\chi^{2}$ (combined with -s): $\chi^{2} \mathrm{~s}(92, \mathrm{~N}=93$ ) substantial part (up to 50\%) of the collected data (SciMag2006)

$>5.19, p \mathrm{~s}<.03($ JPSP2004)

\begin{tabular}{|c|c|c|c|c|c|c|c|c|c|c|c|c|c|c|}
\hline & & & & & & & & & & ciMag2006 & & & & \\
\hline JPSP2004 & SciMag2006 & JESP2008 & PsySci2010 & $M r E-Q_{A S}$ & JPSP2004 & SciMag2006 & JESP2008 & PsySci2010 & $M r E-Q_{S R}$ & JPSP2004 & SciMag2006 & JESP2008 & PsySci2010 & $M r E-Q_{I C}$ \\
\hline 26 & 7 & 5 & 7 & 0.71 & 17 & 15 & 6 & 9 & 0.75 & 21 & 11 & 7 & 11 & 0.79 \\
\hline
\end{tabular}
Sig. effects should have been observed (JPSP2004, PsySci2010
SciMag2006 


\section{Step IV: Reproducibility and Bones' $\Psi$}

The evaluation of issues with individual experiments in Step III also involves an accuracy check of the results and if possible a test of their reproducibility. Ideally, but not necessarily, this check should be based on the original raw data (we analyzed the aggregated data of PsySci2010). It is known however that in many cases data will not be available for post-publication analysis (e.g. Wicherts, Borsboom, Kats, \& Molenaar, 2006), which makes it difficult to notice failures of the expert peerreview to detect errors (e.g., Bakker \& Wicherts, 2011). In most cases it will not be very complicated to evaluate the accuracy of published results based on provided statistics or their reconstruction. One would expect reviewers to perform such basic checks, but in reality authors are allowed (and sometimes asked) to omit important methodological details that would enable a fact-check (Lebel et al., 2013). To show that even the least informative or sub-standard quality result sections allow for a proper review, we describe several reconstructions of statistics and analyses in the SOM (e.g., estimating $n$ in a design cell from a $t$ value and $S D$, or estimating $S E$ measured from a bar graph).

A crucial part of the evaluation is to build a table for each publication in which all the test statistics that are reported in a study, are listed. Table 1 is an excerpt of Table $\mathrm{S} 1$ for the entire core (available in the SOM). It shows that in Experiment 2 of JPSP2004 there is a strange way of reporting results of a $\chi^{2}$-test ("As Published"). The "Effective $\alpha$-level" concerns issues detected with IC. For instance, test number 4 and 7 were reported (without argumentation) as "one-tailed" and the observed $p$ value was halved. This is a serious statistical error, because in this context $\chi^{2}$ is always a right-sided goodness-of-fit test, therefore, the effective $\alpha$ for these tests are listed as 0.10 instead of 0.05 . We introduce a prediction confirmation index, Bones' $\Psi$, quantifying the ability of authors to predict the outcome of experiments as described by Bones (2012). It is the ratio of the significant effects on the total number of evaluated effects. We also calculate Bones' $\Psi$ for the subset of statistics that test core hypotheses identified in Step II. Note that this study reported just one direct test of a core hypothesis (indicated by an asterisk).

Reconstruction will mostly concern a simple (Bonferroni) adjustment of the $\alpha$-level for each family of tests conducted on the study sample. It is important to note that we make the assumption that the studies are confirmatory and not exploratory. In case of the latter, we should not attempt to identify families of tests and adjust the $\alpha$-level for every test conducted. Table 1 shows it was possible to reconstruct two tests (5 and 6) of which the statistics were not disclosed. Also, one reported test statistic (8) was considered invalid (significance test of an unspecified correlation between two dichotomous variables). After reconstruction, Bones' $\Psi$ is much lower and based on these tests the conclusion would be that no evidence for the core hypotheses was observed, one auxiliary hypothesis evidenced more global judgments in UT than in CT.

UTT often predicts null-results (e.g., no effect of problem complexity in unconscious thought conditions). Such predictions are hard to evaluate under NHST, but for fairness and simplicity, we counted a non-significant test as if it were a significant test if it was indeed predicted by UTT, but only when calculating (reconstructed) Bones' $\Psi$. Tests of predicted null results were disregarded when evaluating the Simplest Test of the Hypotheses Possible (STOHP) and hypothesis tests that use effect size confidence intervals (ES-CI; e.g., Cumming, 2012).

\section{Results and Discussion}

\section{What expert peer-review procedure?}

The results of our assessment of the peer-review quality of the core of UTT as indicated by the Marginal results Evaluation Quality are of an unacceptable level across journals. Overall, the four included publications have 2.3 issues associated with each statistic reported. Granted, there is a level of subjectivity in what should be considered an issue, but an inspection of the SR category alone (partial MrE- $Q_{\mathrm{SR}}=0.75$ ) justifies our qualification. The most severe SR issues are with the changing numbers of participants across tests, conducted on the sample of a single study. There may be valid reasons to do this, but they were not reported by the authors or demanded by reviewers. We also have to bring up the 
failure of the random assignment of subjects to conditions: We did not find a single study with a fully balanced design, closest next to PsySci2010 were probably the field studies of SciMag2006 in which there was no random assignment. In the other categories we sincerely believe that a goodness-of-fit test comparing subsets of a design factor for a binary DV is a wrong analysis strategy (it should in fact be considered a public $p$-hack to drop a design level like that). In terms of interpretations, insignificant results were reported as confirmed predictions (JPSP2004 - Experiment 1), or confirmations were based on level comparisons of interaction effects that were not significant at the design level, or not properly tested as an interaction (this is observed throughout the core). Table 2 is a non-exhaustive descriptive summary of issues observed for each category. The bottom row lists next to the number of issues per study the partial MrE-Q per category.

How can high-impact journals maintain they impose a rigorous expert peer review procedure on submitted manuscripts? Certainly, the authors are responsible for the quality of the manuscripts they submit, but in this case, the experts failed in four articles: Who reviews the reviewers? (see Wicherts, Kievit, Bakker, \& Borsboom, 2012 for a case study) We leave it to the reader to draw his or her own conclusions, but to us it is clear that anonymous review serves no purpose in an open and progressive science.

\section{Scientific Credibility of UTT - Predictive Power \& Statistical Power}

The results necessary for an evaluation of the scientific credibility of UTT are presented in Table 3. A clear pattern can be observed across the publications: The original results sections report many tests per study, but no correction for multiple comparisons is used. The need for such adjustments is especially urgent for publications with effective $\alpha$ above 0.05 , indicative of claims of confirmation of a prediction based on insignificant results. The same holds for studies that show signs of $p$-hacking. When omissions and errors are corrected (e.g. family-wise adjustment of $\alpha$ ), the reconstructed results suggest less support for hypotheses, indicated by a lower $\Psi$. The ratio for STOHP $\Psi$ and ES-CI $\Psi$ is generally based on fewer tests, and one or two confirmations could yield a high value. In all cases however, STOHP and ES-CI $\Psi$ indicate a similar drop as the reconstructed $\Psi$. These three different perspectives on hypothesis testing reveal that under close scrutiny, there is a dearth of empirical evidence the core predictions of UTT. This evidential drop-off is depicted in Figure 2. For the whole group of publications, the drop-off is from $92.6 \%$ significant tests, to $36.7 \%$ for reconstructed statistics, to $26.7 \%$ for STOHP and $37.5 \%$ for ES-CI.

Note that a critical deliberator should have arrived at this conclusion without any reconstructions, additional tests or even adjustments of the $\alpha$ level. If we evaluate only the core predictions UT $>\mathrm{CT} \geq \mathrm{IM}$ on quality of choice, but reject evidence based on $p>.05$ (including unjustified level comparisons or directed testing), then on an estimated 1069 participants in 11 studies, 10 out of 23 opportunities for testing one of the nine core predictions provided empirical evidence to support UTT. That is not a robust phenomenon. The more recent publications also affect the logical consistency of the core by invalidating results of previous studies. We make note of such inconsistencies in the three page reports of JESP2008 and PsySci2010. To us this indicates auxiliary hypotheses were proposed in order to save the core phenomena. The last column in Table 3 concerns estimates of empirical accuracy. The procedure for post-hoc achieved power was the same as recently used by Button et al. (2013): The theoretical core was included in SocCog2011, therefore the estimates of achieved power concern the ability to detect the $E S$ reported in $S o c \operatorname{Cog} 2011$. The result is that all the core studies were underpowered (ranging from 0.06 to 0.29 , see SOM for a detailed table). 
EVALUATE BEFORE YOU REPLICATE

\begin{tabular}{|c|c|c|c|c|c|c|c|c|c|c|c|c|c|c|c|c|}
\hline \multirow[b]{2}{*}{ Publication } & \multirow[b]{2}{*}{ Core Hypotheses of the publication } & \multirow[b]{2}{*}{$M r E-Q$} & \multicolumn{4}{|c|}{ As Published } & \multicolumn{4}{|c|}{ Reconstructed } & \multicolumn{4}{|c|}{ STOHР $^{1}$} & \multicolumn{2}{|c|}{ Empirical Accuracy } \\
\hline & & & $\begin{array}{c}\text { Effective } \\
\alpha\end{array}$ & \# Stats. & \# Sig. & Bones' $\Psi$ & $\begin{array}{c}\text { Mean } \\
\alpha\end{array}$ & \# Stats. & \# Sig. & Bones' $\Psi$ & $\begin{array}{c}\text { Mean } \\
\alpha\end{array}$ & \# Stats. & \# Sig. & 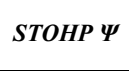 & $\begin{array}{l}\text { Post-hoc } \\
\text { Power }^{2}\end{array}$ & $E S-C I \Psi^{3}$ \\
\hline JPSP2004 & [all tests] & 2.2 & 0.06 & 29 & 22 & 0.76 & 0.02 & 30 & 12 & 0.40 & & & & & 0.16 & 0.24 \\
\hline Study $1-3$ & $\begin{array}{l}\text { UT is superior to IM and CT is inferior } \\
\text { to UT (These hypotheses are tested in all } \\
\text { studies) }\end{array}$ & & 0.07 & 5 & 5 & 1.00 & 0.02 & 6 & 1 & 0.17 & 0.03 & 6 & 1 & 0.17 & 0.13 & 0.17 \\
\hline SciMag2006 & [all tests] & 2.4 & 0.06 & 14 & 11 & 0.79 & 0.03 & 14 & 5 & 0.36 & & & & & 0.12 & 0.36 \\
\hline Study $1-4$ & $\begin{array}{l}\text { Choice in UT is unaffected by } \\
\text { complexity. Choice in CT is worse for } \\
\text { complex problems. }\end{array}$ & & 0.06 & 7 & 6 & 0.86 & 0.02 & 7 & 2 & 0.29 & 0.01 & 9 & 1 & 0.11 & 0.13 & 0.36 \\
\hline JESP2008 & [all tests] & 2.3 & 0.05 & 8 & 5 & 0.63 & 0.03 & 8 & 4 & 0.50 & & & & & 0.25 & 0.67 \\
\hline Study $1 a \& 3$ & $\begin{array}{l}\text { A goal will improve the quality of } \\
\text { decisions in UT. The unconscious obeys } \\
\text { specific goals. }\end{array}$ & & 0.05 & 4 & 4 & 1.00 & 0.03 & 5 & 3 & 0.60 & 0.01 & 7 & 4 & 0.57 & 0.24 & 0.67 \\
\hline PsySci2010 & [all tests] & 2.3 & 0.05 & 9 & 5 & 0.56 & 0.03 & 9 & 2 & 0.22 & & & & & 0.23 & 0.38 \\
\hline $\begin{array}{c}\text { Study } 1 \& 2 \\
\text { (not erratum tests) }\end{array}$ & $\begin{array}{l}\text { UT changes initial impressions. Initial } \\
\text { impressions and choice are unrelated in } \\
\text { UT. }\end{array}$ & & 0.05 & 8 & 7 & 0.88 & 0.03 & 8 & 4 & 0.50 & 0.02 & 7 & 2 & 0.29 & 0.20 & 0.38 \\
\hline PsySci2012 & $\begin{array}{l}\text { A crucial test was corrected to } p<.08 \text {. } \\
\text { The erratum suggests it is marginally } \\
\text { significant. }\end{array}$ & & 0.07 & 3 & 3 & 1.00 & 0.02 & 3 & 1 & 0.33 & 0.02 & 1 & 0 & 0.00 & n.a & 0.00 \\
\hline
\end{tabular}

${ }^{1}$ Note that for both STOHP $\Psi$ and $E S$-CI $\Psi$, the \# of (significant) statistics do not include tests of predicted null-results.

${ }^{2}$ This represents the median achieved power based on overall $E S$ ([all tests] row) and of the moderator $E S$ applicable to the study (prediction row). A detailed table is available in SOM.

${ }^{3}$ This represents Bones' $\Psi$ calculated from tests of the hypotheses based on confidence intervals around the estimated population ES. See SOM for a more detailed table. 


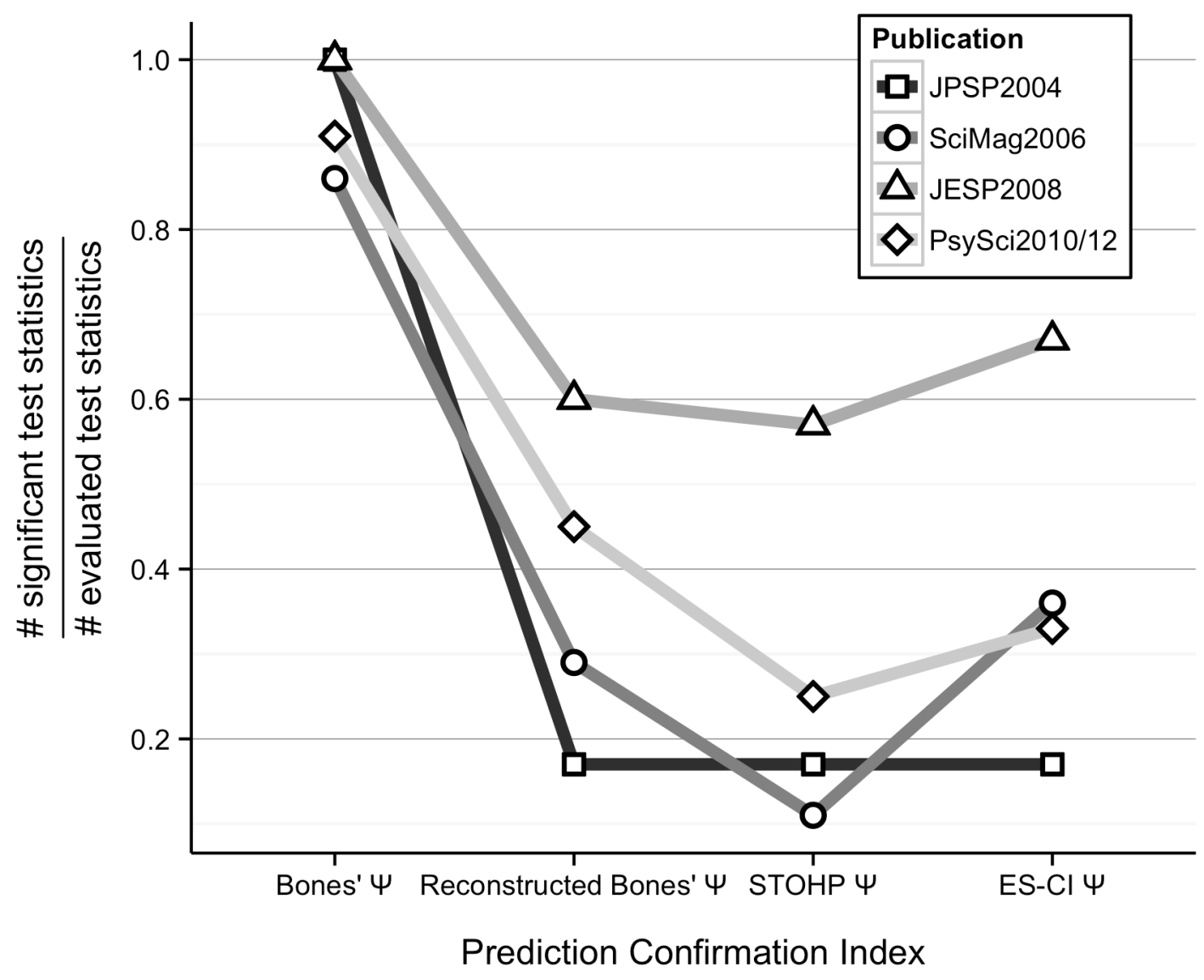

Figure 2. The evidential drop-off of core hypotheses of UTT, for each reviewed publication and index of prediction confirmation. 


\section{General Conclusion: Evaluate before you replicate!}

To be frank, we expected our analysis would enable us to write a paper about theoretical and conceptual issues surrounding UTT. Instead, we encountered a massive failure of expert peer-review to reject manuscripts of a quality unacceptable as a product of science. We conclude the core has been breached and consider UT $=\mathrm{CT}=\mathrm{IM}$.

We do not question the veracity of the data, but we do question the critical stance of authors and reviewers towards UTT and wonder why it's evaluation is dissociated from the larger scientific record of empirical phenomena that describe the unreflective emergence of meaning in participants. A literature review for instance produces findings that make us question how 'up-to-date' the unconscious of UTT really is (cf. Bargh, 2011). The most striking example is a study published in JPSP by Gall and Mendelssohn (1967). They studied facilitation of creative problem solving and two of the three conditions sound familiar: Incubation by distraction (UT) and continued work on the problem (CT). They found CT to be superior over UT for all participants; an effect of experimenter gender on female participants was found in a CT-like condition (CT was 'guided' by association exercises). The report of these results, is of excellent quality and warrants a different experimental setup for UTT studies to control for gender and experimenter effects (cf. Doyen, Klein, Pichon, \& Cleeremans, 2012). In fact, gender differences have been in the empirical record for some time. As reported in the Statistics of "Unconscious Cerebration" (Child, 1892), survey data show the percentage of women below 25 years of age who reported signs of having experienced the merits of "unconscious action" (Child, 1892, p. 463 ) is lower than in men. We estimate $74 \%$ of the participants in the sample we reviewed were female and the majority was younger than 25. Two studies in JPSP2004 report finding the UTE for a small group of males only, which seems consistent with these long known facts.

There is also a 1989 review of incubation studies that examined the effect of participants being "forewarned" they would return to the decision problem or not, after being distracted by another task (Kaplan \& Davidson, 1989). This resembles the studies in JESP2008, in which a "mere distraction" condition was used to argue for the goal-dependency of UT. The conclusion of the 1989 review still appears to apply to the results of JESP2008: Knowledge about returning to the task is not systematically associated with signs of unreflective emergence of meaning in participants. For now, we can only agree with Ireland: "After having carefully considered the evidence upon which the theory of unconscious cerebration is supposed to rest, I am disposed to think that the facts, or assumed facts, may be explained in a simpler manner, and that the theory itself is superfluous and unproved." (Ireland, 1875 , p. 321)

To summarize, our results show it is possible to evaluate a theory of construction by evaluating the track record of the predictive power of its core hypotheses. For UTT, the ability to prescribe a measurement context in which the UTE can be reliably observed is insufficient. The rough estimates of empirical accuracy we used lead to the same conclusion. Given this track record, a potential replication attempt would only be scientifically interesting if some degree of strong inference could be established. For instance, if the study were pre-registered and proponents and opponents agreed in advance on the interpretation of potential outcomes. In science, modesty should accompany laying explanatory claim on a part of reality, particularly when the claim concerns a territory that has been explored so extensively in the history of science. 


\section{References}

Asendorpf, J. B., Conner, M., de Fruyt, F., de Houwer, J., Denissen, J. J. A., Fiedler, K., Fiedler, S., et al. (2013). Recommendations for increasing replicability in psychology. European Journal of Personality, 27, 108-119. doi:10.1002/per.1919

Bakker, M., \& Wicherts, J. M. (2011). The (mis)reporting of statistical results in psychology journals. Behavior Research Methods, 43, 666-78. doi:10.3758/s13428-011-0089-5

Bargh, J. A. (2011). Unconscious Thought Theory and its discontents: A critique of the critiques. Social Cognition, 29, 629-647. doi:10.1521/soco.2011.29.6.629

Bokulich, A. (2004). Open or closed? Dirac, Heisenberg, and the relation between classical and quantum mechanics. Studies in History and Philosophy of Modern Physics, 35, 377-396. doi:10.1016/j.shpsb.2003.11.002

Bones, A. K. (2012). We knew the future all along: Scientific hypothesizing is much more accurate than other forms of precognition--a satire in one part. Perspectives on Psychological Science, 7 , 307-309. doi:10.1177/1745691612441216

Bos, M. W., Dijksterhuis, A., \& van Baaren, R. B. (2008). On the goal-dependency of unconscious thought. Journal of Experimental Social Psychology, 44, 1114-1120. doi:10.1016/j.jesp.2008.01.001

Bosman, A.M.T., Cox, R.F.A., Hasselman, F., \& Wijnants, M.L. (in press). From the role of context to the measurement problem: The Dutch connection pays tribute to Guy Van Orden. Ecological Psychology. DOI:10.1080/10407413.2013.810091

Browne, J. H. B. (1870). On the method of the study of mind. The British Journal of Psychiatry, 16(74), 233-247. doi:10.1192/bjp.16.74.233

Button, K. S., Ioannidis, J. P. A., Mokrysz, C., Nosek, B. A., Flint, J., Robinson, E. S. J., \& Munafò, M. R. (2013). Power failure: why small sample size undermines the reliability of neuroscience. Nature Reviews Neuroscience, 14(May). doi:10.1038/nrn3475

Child, C. (1892). Statistics of "unconscious cerebration". The American Journal of Psychology, 5, 249259. Retrieved from http://www.jstor.org/stable/10.2307/1410868

Cumming, G. (2012). Understanding the new statistics: Effect sizes, confidence intervals, and metaanalysis. NY: Routledge.

Dijksterhuis, A. (2004). Think different: The merits of unconscious thought in preference development and decision making. Journal of Personality and Social Psychology, 87, 586-98. doi:10.1037/0022-3514.87.5.586

Dijksterhuis, A., Bos, M. W., Nordgren, L. F., \& van Baaren, R. B. (2006a). On making the right choice: the deliberation-without-attention effect. Science, 311(5763), 1005-7. doi:10.1126/science. 1121629 
Dijksterhuis, A., Bos, M. W., Nordgren, L. F., \& van Baaren, R. B. (2006b). Supporting online material for: On making the right choice: the deliberation-without-attention effect. Science, 311(5763), 1005-7. doi:10.1126/science. 1121629

Dijksterhuis, A., \& Nordgren, L. F. (2006). A theory of unconscious thought. Perspectives on Psychological Science, 1, 95-109. doi:10.1111/j.1745-6916.2006.00007.x

Doyen, S., Klein, O., Pichon, C.-L., \& Cleeremans, A. (2012). Behavioral priming: It's all in the mind, but whose mind? PLoS ONE, 7(1), e29081. doi:10.1371/journal.pone.0029081

Elms, A. (1975). The crisis of confidence in social psychology. American Psychologist, (October), 967-976. Retrieved from http://psycnet.apa.org/psycinfo/1976-04477-001

Fanelli, D. (2010). "Positive" results increase down the Hierarchy of the Sciences. PLoS ONE, 5(4), e10068. doi:10.1371/journal.pone.0010068

Fiedler, K., Kutzner, F., \& Krueger, J. I. (2012). The long way from $\alpha$-error control to validity proper: Problems with a short-sighted false-positive debate . Perspectives on Psychological Science , 7 , 661-669. doi:10.1177/1745691612462587

Gall, M., \& Mendelsohn, G. A. (1967). Effects of facilitating techniques and subject-experimenter interaction on creative problem solving. Journal of Personality and Social Psychology, 5, 211-6. doi: $10.1037 / \mathrm{h} 0024130$

Heisenberg, W. (1963). Oral history interview of Werner Heisenberg by Thomas Kuhn. Archive for the History of Quantum Physics, deposit at Harvard University. Cambridge, MA.

Hogg, M. A., \& Grieve, P. (1999). Social identity theory and the crisis of confidence in social psychology: A commentary, and some research on uncertainty reduction. Asian Journal of Social Psychology, 2, 79-93. doi:10.1111/1467-839X.00027

Ioannidis, J. P. A. (2012). Why science is not necessarily self-correcting. Perspectives on Psychological Science, 7, 645-654. doi:10.1177/1745691612464056

Ireland, W. W. (1875). Can unconscious cerebration be proved? The British Journal of Psychiatry, 21(95), 366-387. doi:10.1192/bjp.21.95.366

Kaplan, C. A., \& Davidson, J. (1989). Hatching a theory of incubation effects (No. AIP-98). Pittsburgh, PA: Carnegie-Mellon University of Pittsburgh, Artifical Intelligence and Psychology Project. Retrieved from http://oai.dtic.mil/oai/oai?verb=getRecord\&metadataPrefix=html\&identifier=ADA219275

Ladd, G. (1892). Psychology as so-called "natural science". The Philosophical Review, 1(1), 24-53. Retrieved from http://www.jstor.org/stable/10.2307/2175528

Lakatos, I. (1976). Proofs and refutations: the logic of mathematical discovery (p. xii, 174 p.). Cambridge, NY: Cambridge University Press. 
LeBel, E. P., Borsboom, D., Giner-sorolla, R., Hasselman, F., Peters, K. R., Ratliff, K. A., \& Tucker Smith, C. (2013). PsychDisclosure.org: Grassroot Support for Reforming Reporting Standards in Psychology Perspectives on Psychological Science, in press, 1-10.

LeBel, E. P., \& Peters, K. R. (2011). Fearing the future of empirical psychology: Bem's (2011) evidence of psi as a case study of deficiencies in modal research practice. Review of General Psychology, 15(4), 371-379. doi:10.1037/a0025172

Makel, M. C., Plucker, J. A., \& Hegarty, B. (2012). Replications in psychology research: How often do they really occur? Perspectives on Psychological Science, 7, 537-542. doi: $10.1177 / 1745691612460688$

Molenaar, P. C. M. (2008). On the implications of the classical ergodic theorems: Analysis of developmental processes has to focus on intra-individual variation. Developmental Psychobiology, 50, 60-69. doi:10.1002/dev

Open Science Collaboration. (2012). An open, large-scale, collaborative effort to estimate the reproducibility of psychological science. Perspectives on Psychological Science, 7, 657-660. doi: $10.1177 / 1745691612462588$

Pashler, H., \& Wagenmakers, E.-J. (2012). Editors' introduction to the special section on replicability in psychological science: A crisis of confidence? Perspectives on Psychological Science, 7, 528530. doi:10.1177/1745691612465253

Platt, J. (1964). Strong Inference: Certain systematic methods of scientific thinking may produce much more rapid progress than others. Science, 146(3642), 347-353. doi:10.1126/science.146.3642.347

Rietveld, E. (2008). Special Section: The Skillful Body as a Concernful System of Possible Actions: Phenomena and Neurodynamics. Theory \& Psychology, 18(3), 341-363. doi:10.1177/0959354308089789

Simmons, J. P., Nelson, L. D., \& Simonsohn, U. (2011). False-positive psychology: Undisclosed flexibility in data collection and analysis allows presenting anything as significant. Psychological Science, 22, 1359-66. doi:10.1177/0956797611417632

Sio, U. N., \& Ormerod, T. C. (2009). Does incubation enhance problem solving? A meta-analytic review. Psychological Bulletin, 135, 94-120. doi:10.1037/a0014212

Spencer, H. (1855). The principles of psychology. London, UK: Longman, Brown, Green and Longmans. Retrieved from http://oll.libertyfund.org/title/1394

Stephen, D. G., Dixon, J. A., \& Isenhower, R. W. (2009). Dynamics of representational change: Entropy, action and cognition. Journal of Experimental Psychology: Human Perception and Performance, 35, 1811-1832. doi: 10.1037/a0014510

Stickgold, R., \& Walker, M. (2004). To sleep, perchance to gain creative insight? Trends in Cognitive Sciences, 8, 191-192. doi:10.1016/j.tics.2004.03.003 
Strick, M., Dijksterhuis, A., Bos, M. W., Sjoerdsma, A., van Baaren, R. B., \& Nordgren, L. F. (2011). A meta-analysis on unconscious thought effects. Social Cognition, 29, 738-762. doi:10.1521/soco.2011.29.6.738

Strick, M., Dijksterhuis, A., \& van Baaren, R. B. (2010). Unconscious-thought effects take place offline, not on-line. Psychological Science, 21, 484-488. doi:10.1177/0956797610363555

Strick, M., Dijksterhuis, A., \& van Baaren, R. B. (2012). Erratum. Psychological Science, 23, 109. doi: $10.1177 / 0956797611432836$

van Dongen, J. (2010). Einstein's unification. Cambridge, NY: Cambridge University Press.

Vul, E., \& Pashler, H. (2012). Voodoo and circularity errors. NeuroImage, 62, 945-948. doi:10.1016/j.neuroimage.2012.01.027

Waroquier, L., Marchiori, D., Klein, O., \& Cleeremans, A. (2009). Methodological pitfalls of the Unconscious Thought paradigm. Judgment and Decision Making, 4, 601-610. doi:??

Wicherts, J. M., Borsboom, D., Kats, J., \& Molenaar, D. (2006). The poor availability of psychological research data for reanalysis. The American psychologist, 61, 726-728. doi:10.1037/0003066X.61.7.726

Wicherts, J. M., Kievit, R. A, Bakker, M., \& Borsboom, D. (2012). Letting the daylight in: Reviewing the reviewers and other ways to maximize transparency in science. Frontiers in Computational Neuroscience, 6:20. doi:10.3389/fncom.2012.00020

Wong, P. (1981). Implicit editorial policies and the integrity of psychology as an empirical science. American Psychologist, 36, 690-691. doi:10.1037/0003-066X.36.6.690

Woodworth, R. S., \& Schlosberg, H. (1954). Experimental psychology. NY: Chicago: Holt, Rinehart and Winston. 
EVALUATE BEFORE YOU REPLICATE

Table 1

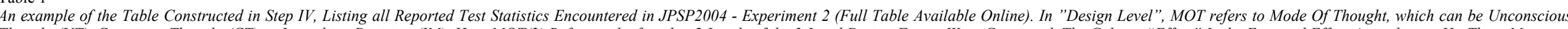

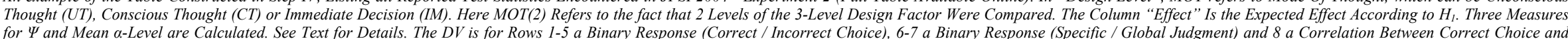
Specific / Global Judgment.

\begin{tabular}{|c|c|c|c|c|c|c|c|c|c|c|c|c|c|c|c|}
\hline \# & Design Level & Effect & As published & $p$ & Effective $\alpha$ & Reject $\mathbf{H}_{0}$ & Recon. Stats & $p$ & Family & Recon. $\alpha$ & Reject $\mathbf{H}_{0}$ & STOHP & $p$ & Adjusted $\alpha$ & Reject $\mathbf{H}_{0}$ \\
\hline 1 & IM & "Better than chance" & $\chi^{2} \mathrm{~s}(92, N=93)>5.19$ & 0.0300 & 0.0500 & YES & $\chi^{2} \mathrm{~s}(1)>5.19$ & ? & $\mathrm{A} 1$ & 0.0167 & - & & & & \\
\hline 2 & СТ & "Better than chance" & $\chi^{2} \mathrm{~s}(92, N=93)>5.19$ & 0.0300 & 0.0500 & YES & $\chi^{2} \mathrm{~s}(1)>5.19$ & $?$ & A2 & 0.0167 & - & & & & \\
\hline 3 & UT & "Better than chance" & $\chi^{2} \mathrm{~s}(92, N=93)>5.19$ & 0.0300 & 0.0500 & YES & $\chi^{2} \mathrm{~s}(1)>5.19$ & ? & A3 & 0.0167 & - & & & & \\
\hline 4 & $\operatorname{MOT}(2)$ & $\mathrm{UT}>\mathrm{IM}$ & $\chi^{2}(59, N=60)=3.13^{*}$ & 0.0770 & 0.1000 & YES* & $\chi^{2}(1)=3.13^{*}$ & 0.0770 & B1 & 0.0250 & - & $Z=1.77^{*}$ & 0.0770 & 0.0250 & - \\
\hline 5 & MOT(2) & $\mathrm{UT}>\mathrm{CT}$ & ? & $?$ & ? & - & $\chi^{2}(1)=0.90^{*}$ & 0.3433 & B2 & 0.0250 & - & $Z=0.95^{*}$ & 0.3430 & 0.0250 & - \\
\hline 6 & $\operatorname{MOT}(2)$ & $\mathrm{UT}>\mathrm{IM}$ & $?$ & $?$ & ? & - & $\chi^{2}(1)=1.03$ & 0.3112 & $\mathrm{C} 1$ & 0.0250 & - & & & & \\
\hline 7 & MOT(2) & $\mathrm{UT}>\mathrm{CT}$ & $\chi^{2}(60, N=61)=6.69$ & 0.0098 & 0.1000 & YES & $\chi^{2}(1)=5.34$ & 0.0209 & $\mathrm{C} 2$ & 0.0250 & YES & & & & \\
\hline \multirow[t]{4}{*}{8} & Sample? & $r>0$ & $r=0.22$ & 0.0400 & 0.0500 & YES & ? & ? & ? & ? & - & & & & \\
\hline & & & \multicolumn{2}{|c|}{ Mean $\alpha$-level: } & 0.0667 & & & & Mean $\alpha$-level: & 0.0214 & & \multicolumn{2}{|c|}{ Mean $\alpha$-level: } & 0.0250 & \\
\hline & & & \multicolumn{2}{|c|}{ All Tests - Bones' $\Psi$} & 1.00 & $6 / 6$ & \multicolumn{3}{|c|}{ All Tests - Reconstructed Bones' $\Psi$} & 0.14 & $1 / 7$ & & & & \\
\hline & & & \multicolumn{2}{|c|}{ Tests of Hypotheses - Bones' $\Psi$} & 1.00 & $1 / 1$ & \multicolumn{3}{|c|}{ Tests of Hypotheses - Reconstructed Bones' $\Psi$} & 0.00 & $0 / 2$ & & 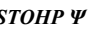 & 0.00 & $0 / 2$ \\
\hline
\end{tabular}




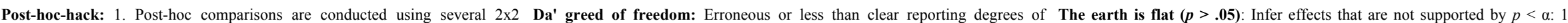
ANOVAs (JPSP2004, SciMag2006, JESP2008,) 2. No adjustments for freedom as evidenced by the test statistic and / or $n$ that are reported for a Based on $p<.08$ or n.s. (JPSP2004, PsySci2012, SocCog2011). 2. Observed $\chi$ multiple comparisons of the $\alpha$-level are reported (JPSP2004, SciMag2006, design cell (JPSP2004, SciMag2006, JESP2008, PsySci2010)
JESP2008, PsySci2010) 3. Separate Sig. F-tests (1 or 2 main, interaction) reported whereas evaluation is straightforward at the level of the full model (JPSP2004, SciMag2006, JESP2008,) hoc effects would be insignificant if $\alpha$ were adjusted for multiple comparison (JPSP2004, JESP2008, SciMag2006, PsySci2010)

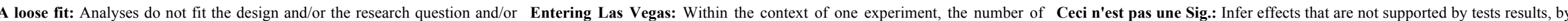
the measured variables: 1 . Use $\chi$ as if it were a model with a linear predictor paticipants in design cells appeans to change from analysis to analysis. These using test results that support a different effect (related to public $p$-hacking): 1 .

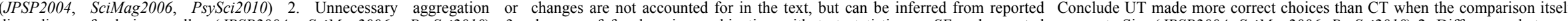

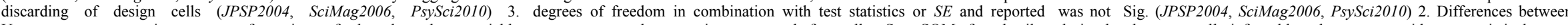

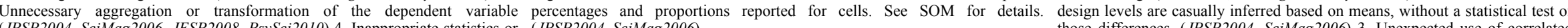
tests (JPSP2004, SciMag2006, PsySci2010) DVs or factors to suggest a core effect was at work (JPSP2004, SciMag2006, PsySci2010). 4. In JESP2008 the predicted effect is Sig. only for one stimulus set. The prediction is still reported as confirmed, consequences for previous
studies were not discussed.

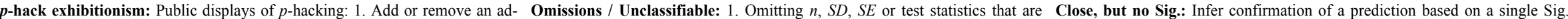

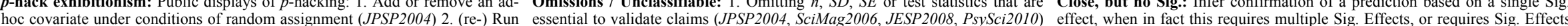

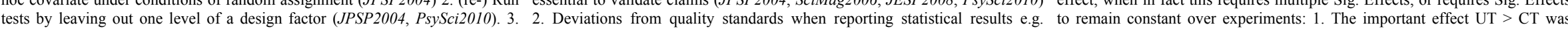

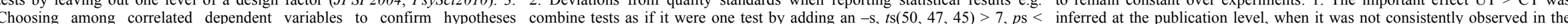
(JPSP2004, PsySci2010) 4. Post-data collection exclusion rule used to discard a .02 ; unknown format for (wrong) $d f$ of $\chi^{2}$ (combined with -s): $\chi^{2} \mathrm{~s}(92, \mathrm{~N}=93$ ) substantial part (up to 50\%) of the collected data (SciMag2006)

$$
>5.19, p \mathrm{~s}<.03(\text { (JPSP2004) }
$$

\begin{tabular}{|c|c|c|c|c|c|c|c|c|c|c|c|c|c|c|}
\hline & & & & & & & & & & ciMag2006 & & & & \\
\hline JPSP2004 & SciMag2006 & JESP2008 & PsySci2010 & $M r E-Q_{A S}$ & JPSP2004 & SciMag2006 & JESP2008 & PsyScizo10 & $M r E-Q_{S R}$ & JPSP2004 & SciMag2006 & JESP2008 & PsySci2010 & $M r E-Q_{I C}$ \\
\hline 26 & 7 & 5 & 7 & 0.71 & 17 & 15 & 6 & 9 & 0.75 & 21 & 11 & 7 & 11 & 0.79 \\
\hline
\end{tabular}




\section{Step IV: Reproducibility and Bones' $\Psi$}

The evaluation of issues with individual experiments in Step III also involves an accuracy check of the results and if possible a test of their reproducibility. Ideally, but not necessarily, this check should be based on the original raw data (we analyzed the aggregated data of PsySci2010). It is known however that in many cases data will not be available for post-publication analysis (e.g. Wicherts, Borsboom, Kats, \& Molenaar, 2006), which makes it difficult to notice failures of the expert peerreview to detect errors (e.g., Bakker \& Wicherts, 2011). In most cases it will not be very complicated to evaluate the accuracy of published results based on provided statistics or their reconstruction. One would expect reviewers to perform such basic checks, but in reality authors are allowed (and sometimes asked) to omit important methodological details that would enable a fact-check (Lebel et al., 2013). To show that even the least informative or sub-standard quality result sections allow for a proper review, we describe several reconstructions of statistics and analyses in the SOM (e.g., estimating $n$ in a design cell from a $t$ value and $S D$, or estimating $S E$ measured from a bar graph).

A crucial part of the evaluation is to build a table for each publication in which all the test statistics that are reported in a study, are listed. Table 1 is an excerpt of Table $\mathrm{S} 1$ for the entire core (available in the SOM). It shows that in Experiment 2 of JPSP2004 there is a strange way of reporting results of a $\chi^{2}$-test ("As Published"). The "Effective $\alpha$-level" concerns issues detected with IC. For instance, test number 4 and 7 were reported (without argumentation) as "one-tailed" and the observed $p$ value was halved. This is a serious statistical error, because in this context $\chi^{2}$ is always a right-sided goodness-of-fit test, therefore, the effective $\alpha$ for these tests are listed as 0.10 instead of 0.05 . We introduce a prediction confirmation index, Bones' $\Psi$, quantifying the ability of authors to predict the outcome of experiments as described by Bones (2012). It is the ratio of the significant effects on the total number of evaluated effects. We also calculate Bones' $\Psi$ for the subset of statistics that test core hypotheses identified in Step II. Note that this study reported just one direct test of a core hypothesis (indicated by an asterisk).

Reconstruction will mostly concern a simple (Bonferroni) adjustment of the $\alpha$-level for each family of tests conducted on the study sample. It is important to note that we make the assumption that the studies are confirmatory and not exploratory. In case of the latter, we should not attempt to identify families of tests and adjust the $\alpha$-level for every test conducted. Table 1 shows it was possible to reconstruct two tests (5 and 6) of which the statistics were not disclosed. Also, one reported test statistic (8) was considered invalid (significance test of an unspecified correlation between two dichotomous variables). After reconstruction, Bones' $\Psi$ is much lower and based on these tests the conclusion would be that no evidence for the core hypotheses was observed, one auxiliary hypothesis evidenced more global judgments in UT than in CT.

UTT often predicts null-results (e.g., no effect of problem complexity in unconscious thought conditions). Such predictions are hard to evaluate under NHST, but for fairness and simplicity, we counted a non-significant test as if it were a significant test if it was indeed predicted by UTT, but only when calculating (reconstructed) Bones' $\Psi$. Tests of predicted null results were disregarded when evaluating the Simplest Test of the Hypotheses Possible (STOHP) and hypothesis tests that use effect size confidence intervals (ES-CI; e.g., Cumming, 2012).

\section{Results and Discussion}

\section{What expert peer-review procedure?}

The results of our assessment of the peer-review quality of the core of UTT as indicated by the Marginal results Evaluation Quality are of an unacceptable level across journals. Overall, the four included publications have 2.3 issues associated with each statistic reported. Granted, there is a level of subjectivity in what should be considered an issue, but an inspection of the SR category alone (partial MrE- $Q_{\mathrm{SR}}=0.75$ ) justifies our qualification. The most severe SR issues are with the changing numbers of participants across tests, conducted on the sample of a single study. There may be valid reasons to do this, but they were not reported by the authors or demanded by reviewers. We also have to bring up the 
failure of the random assignment of subjects to conditions: We did not find a single study with a fully balanced design, closest next to PsySci2010 were probably the field studies of SciMag2006 in which there was no random assignment. In the other categories we sincerely believe that a goodness-of-fit test comparing subsets of a design factor for a binary DV is a wrong analysis strategy (it should in fact be considered a public $p$-hack to drop a design level like that). In terms of interpretations, insignificant results were reported as confirmed predictions (JPSP2004 - Experiment 1), or confirmations were based on level comparisons of interaction effects that were not significant at the design level, or not properly tested as an interaction (this is observed throughout the core). Table 2 is a non-exhaustive descriptive summary of issues observed for each category. The bottom row lists next to the number of issues per study the partial MrE-Q per category.

How can high-impact journals maintain they impose a rigorous expert peer review procedure on submitted manuscripts? Certainly, the authors are responsible for the quality of the manuscripts they submit, but in this case, the experts failed in four articles: Who reviews the reviewers? (see Wicherts, Kievit, Bakker, \& Borsboom, 2012 for a case study) We leave it to the reader to draw his or her own conclusions, but to us it is clear that anonymous review serves no purpose in an open and progressive science.

\section{Scientific Credibility of UTT - Predictive Power \& Statistical Power}

The results necessary for an evaluation of the scientific credibility of UTT are presented in Table 3. A clear pattern can be observed across the publications: The original results sections report many tests per study, but no correction for multiple comparisons is used. The need for such adjustments is especially urgent for publications with effective $\alpha$ above 0.05 , indicative of claims of confirmation of a prediction based on insignificant results. The same holds for studies that show signs of $p$-hacking. When omissions and errors are corrected (e.g. family-wise adjustment of $\alpha$ ), the reconstructed results suggest less support for hypotheses, indicated by a lower $\Psi$. The ratio for STOHP $\Psi$ and ES-CI $\Psi$ is generally based on fewer tests, and one or two confirmations could yield a high value. In all cases however, STOHP and ES-CI $\Psi$ indicate a similar drop as the reconstructed $\Psi$. These three different perspectives on hypothesis testing reveal that under close scrutiny, there is a dearth of empirical evidence the core predictions of UTT. This evidential drop-off is depicted in Figure 2. For the whole group of publications, the drop-off is from $92.6 \%$ significant tests, to $36.7 \%$ for reconstructed statistics, to $26.7 \%$ for STOHP and $37.5 \%$ for ES-CI.

Note that a critical deliberator should have arrived at this conclusion without any reconstructions, additional tests or even adjustments of the $\alpha$ level. If we evaluate only the core predictions UT $>\mathrm{CT} \geq \mathrm{IM}$ on quality of choice, but reject evidence based on $p>.05$ (including unjustified level comparisons or directed testing), then on an estimated 1069 participants in 11 studies, 10 out of 23 opportunities for testing one of the nine core predictions provided empirical evidence to support UTT. That is not a robust phenomenon. The more recent publications also affect the logical consistency of the core by invalidating results of previous studies. We make note of such inconsistencies in the three page reports of JESP2008 and PsySci2010. To us this indicates auxiliary hypotheses were proposed in order to save the core phenomena. The last column in Table 3 concerns estimates of empirical accuracy. The procedure for post-hoc achieved power was the same as recently used by Button et al. (2013): The theoretical core was included in SocCog2011, therefore the estimates of achieved power concern the ability to detect the $E S$ reported in $S o c \operatorname{Cog} 2011$. The result is that all the core studies were underpowered (ranging from 0.06 to 0.29 , see SOM for a detailed table). 
EVALUATE BEFORE YOU REPLICATE

\begin{tabular}{|c|c|c|c|c|c|c|c|c|c|c|c|c|c|c|c|c|}
\hline \multirow[b]{2}{*}{ Publication } & \multirow[b]{2}{*}{ Core Hypotheses of the publication } & \multirow[b]{2}{*}{$M r E-Q$} & \multicolumn{4}{|c|}{ As Published } & \multicolumn{4}{|c|}{ Reconstructed } & \multicolumn{4}{|c|}{ STOHP $^{1}$} & \multicolumn{2}{|c|}{ Empirical Accuracy } \\
\hline & & & $\begin{array}{c}\text { Effective } \\
\qquad \alpha\end{array}$ & \# Stats. & \# Sig. & 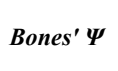 & $\begin{array}{c}\text { Mean } \\
\boldsymbol{\alpha}\end{array}$ & \# Stats. & \# Sig. & 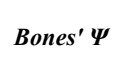 & $\begin{array}{c}\text { Mean } \\
\boldsymbol{\alpha}\end{array}$ & \# Stats. & \# Sig. & STOHP $\Psi$ & $\begin{array}{l}\text { Post-hoc } \\
\text { Power }^{2}\end{array}$ & $E S-C I \Psi^{3}$ \\
\hline JPSP2004 & [all tests] & 2.2 & 0.06 & 29 & 22 & 0.76 & 0.02 & 30 & 12 & 0.40 & & & & & 0.16 & 0.24 \\
\hline Study 1-3 & $\begin{array}{l}\text { UT is superior to IM and CT is inferior } \\
\text { to UT (These hypotheses are tested in all } \\
\text { studies) }\end{array}$ & & 0.07 & 5 & 5 & 1.00 & 0.02 & 6 & 1 & 0.17 & 0.03 & 6 & 1 & 0.17 & 0.13 & 0.17 \\
\hline SciMag2006 & [all tests] & 2.4 & 0.06 & 14 & 11 & 0.79 & 0.03 & 14 & 5 & 0.36 & & & & & 0.12 & 0.36 \\
\hline Study $1-4$ & $\begin{array}{l}\text { Choice in UT is unaffected by } \\
\text { complexity. Choice in CT is worse for } \\
\text { complex problems. }\end{array}$ & & 0.06 & 7 & 6 & 0.86 & 0.02 & 7 & 2 & 0.29 & 0.01 & 9 & 1 & 0.11 & 0.13 & 0.36 \\
\hline JESP2008 & [all tests] & 2.3 & 0.05 & 8 & 5 & 0.63 & 0.03 & 8 & 4 & 0.50 & & & & & 0.25 & 0.67 \\
\hline Study $1 a \& 3$ & $\begin{array}{l}\text { A goal will improve the quality of } \\
\text { decisions in UT. The unconscious obeys } \\
\text { specific goals. }\end{array}$ & & 0.05 & 4 & 4 & 1.00 & 0.03 & 5 & 3 & 0.60 & 0.01 & 7 & 4 & 0.57 & 0.24 & 0.67 \\
\hline PsySci2010 & [all tests] & 2.3 & 0.05 & 9 & 5 & 0.56 & 0.03 & 9 & 2 & 0.22 & & & & & 0.23 & 0.38 \\
\hline $\begin{array}{l}\text { Study } 1 \& 2 \\
\text { (not erratum tests) }\end{array}$ & $\begin{array}{l}\text { UT changes initial impressions. Initial } \\
\text { impressions and choice are unrelated in } \\
\text { UT. }\end{array}$ & & 0.05 & 8 & 7 & 0.88 & 0.03 & 8 & 4 & 0.50 & 0.02 & 7 & 2 & 0.29 & 0.20 & 0.38 \\
\hline PsySci2012 & $\begin{array}{l}\text { A crucial test was corrected to } p<.08 \text {. } \\
\text { The erratum suggests it is marginally } \\
\text { significant. }\end{array}$ & & 0.07 & 3 & 3 & 1.00 & 0.02 & 3 & 1 & 0.33 & 0.02 & 1 & 0 & 0.00 & n.a & 0.00 \\
\hline
\end{tabular}

Note that for both STOHP $\Psi$ and $E S$-CI $\Psi$, the \# of (significant) statistics do not include tests of predicted null-results.

${ }^{2}$ This represents the median achieved power based on overall $E S$ ([all tests] row) and of the moderator $E S$ applicable to the study (prediction row). A detailed table is available in SOM.

${ }^{3}$ This represents Bones' $\Psi$ calculated from tests of the hypotheses based on confidence intervals around the estimated population $E S$. See SOM for a more detailed table. 


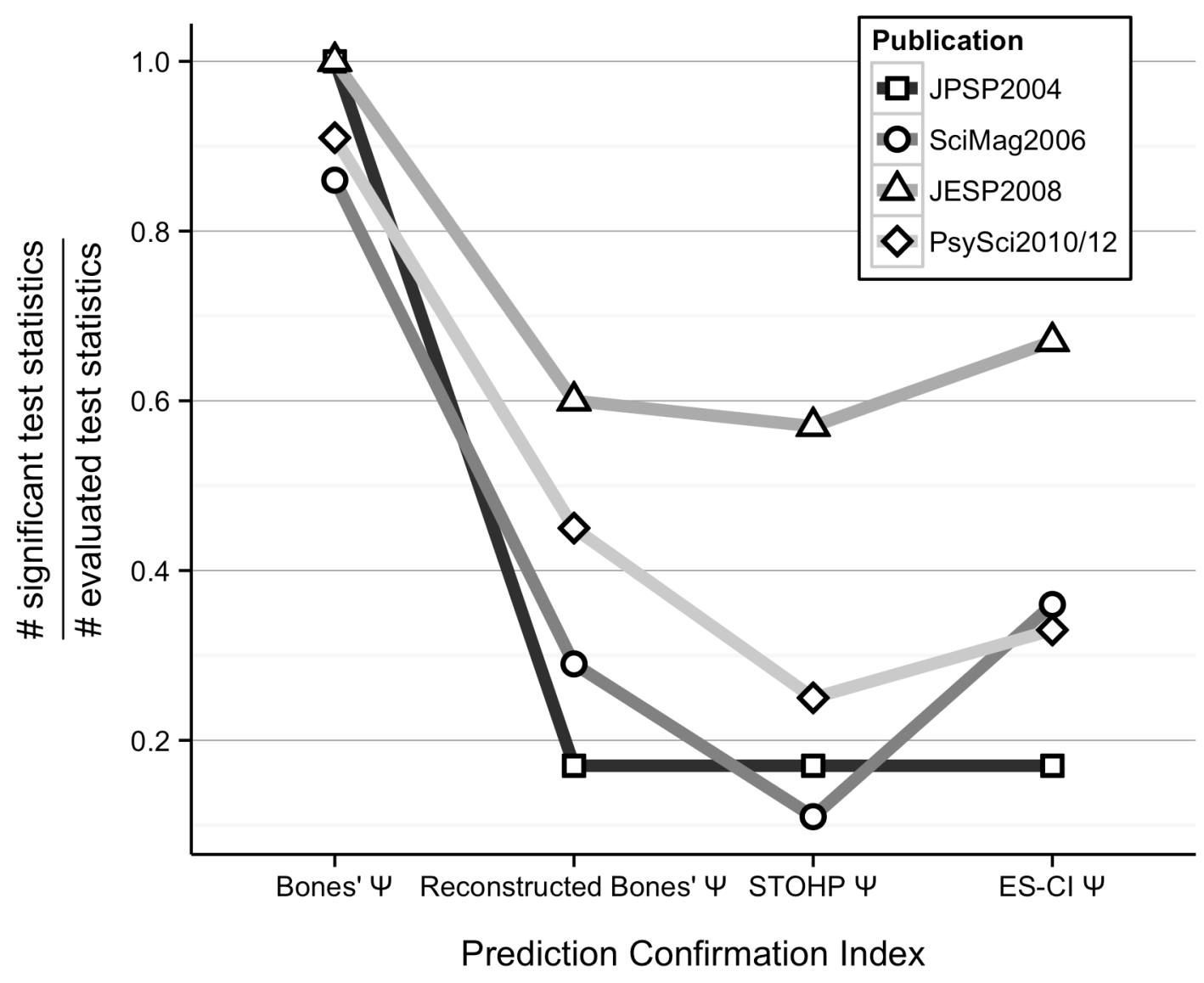

Figure 2. The evidential drop-off of core hypotheses of UTT, for each reviewed publication and index of prediction confirmation. 


\section{General Conclusion: Evaluate before you replicate!}

To be frank, we expected our analysis would enable us to write a paper about theoretical and conceptual issues surrounding UTT. Instead, we encountered a massive failure of expert peer-review to reject manuscripts of a quality unacceptable as a product of science. We conclude the core has been breached and consider UT $=\mathrm{CT}=\mathrm{IM}$.

We do not question the veracity of the data, but we do question the critical stance of authors and reviewers towards UTT and wonder why it's evaluation is dissociated from the larger scientific record of empirical phenomena that describe the unreflective emergence of meaning in participants. A literature review for instance produces findings that make us question how 'up-to-date' the unconscious of UTT really is (cf. Bargh, 2011). The most striking example is a study published in JPSP by Gall and Mendelssohn (1967). They studied facilitation of creative problem solving and two of the three conditions sound familiar: Incubation by distraction (UT) and continued work on the problem (CT). They found CT to be superior over UT for all participants; an effect of experimenter gender on female participants was found in a CT-like condition (CT was 'guided' by association exercises). The report of these results, is of excellent quality and warrants a different experimental setup for UTT studies to control for gender and experimenter effects (cf. Doyen, Klein, Pichon, \& Cleeremans, 2012). In fact, gender differences have been in the empirical record for some time. As reported in the Statistics of "Unconscious Cerebration" (Child, 1892), survey data show the percentage of women below 25 years of age who reported signs of having experienced the merits of "unconscious action" (Child, 1892, p. 463 ) is lower than in men. We estimate $74 \%$ of the participants in the sample we reviewed were female and the majority was younger than 25. Two studies in JPSP2004 report finding the UTE for a small group of males only, which seems consistent with these long known facts.

There is also a 1989 review of incubation studies that examined the effect of participants being "forewarned" they would return to the decision problem or not, after being distracted by another task (Kaplan \& Davidson, 1989). This resembles the studies in JESP2008, in which a "mere distraction" condition was used to argue for the goal-dependency of UT. The conclusion of the 1989 review still appears to apply to the results of JESP2008: Knowledge about returning to the task is not systematically associated with signs of unreflective emergence of meaning in participants. For now, we can only agree with Ireland: "After having carefully considered the evidence upon which the theory of unconscious cerebration is supposed to rest, I am disposed to think that the facts, or assumed facts, may be explained in a simpler manner, and that the theory itself is superfluous and unproved." (Ireland, 1875 , p. 321)

To summarize, our results show it is possible to evaluate a theory of construction by evaluating the track record of the predictive power of its core hypotheses. For UTT, the ability to prescribe a measurement context in which the UTE can be reliably observed is insufficient. The rough estimates of empirical accuracy we used lead to the same conclusion. Given this track record, a potential replication attempt would only be scientifically interesting if some degree of strong inference could be established. For instance, if the study were pre-registered and proponents and opponents agreed in advance on the interpretation of potential outcomes. In science, modesty should accompany laying explanatory claim on a part of reality, particularly when the claim concerns a territory that has been explored so extensively in the history of science. 


\section{References}

Asendorpf, J. B., Conner, M., de Fruyt, F., de Houwer, J., Denissen, J. J. A., Fiedler, K., Fiedler, S., et al. (2013). Recommendations for increasing replicability in psychology. European Journal of Personality, 27, 108-119. doi:10.1002/per.1919

Bakker, M., \& Wicherts, J. M. (2011). The (mis)reporting of statistical results in psychology journals. Behavior Research Methods, 43, 666-78. doi:10.3758/s13428-011-0089-5

Bargh, J. A. (2011). Unconscious Thought Theory and its discontents: A critique of the critiques. Social Cognition, 29, 629-647. doi:10.1521/soco.2011.29.6.629

Bokulich, A. (2004). Open or closed? Dirac, Heisenberg, and the relation between classical and quantum mechanics. Studies in History and Philosophy of Modern Physics, 35, 377-396. doi:10.1016/j.shpsb.2003.11.002

Bones, A. K. (2012). We knew the future all along: Scientific hypothesizing is much more accurate than other forms of precognition--a satire in one part. Perspectives on Psychological Science, 7 , 307-309. doi:10.1177/1745691612441216

Bos, M. W., Dijksterhuis, A., \& van Baaren, R. B. (2008). On the goal-dependency of unconscious thought. Journal of Experimental Social Psychology, 44, 1114-1120. doi:10.1016/j.jesp.2008.01.001

Bosman, A.M.T., Cox, R.F.A., Hasselman, F., \& Wijnants, M.L. (in press). From the role of context to the measurement problem: The Dutch connection pays tribute to Guy Van Orden. Ecological Psychology. DOI:10.1080/10407413.2013.810091

Browne, J. H. B. (1870). On the method of the study of mind. The British Journal of Psychiatry, 16(74), 233-247. doi:10.1192/bjp.16.74.233

Button, K. S., Ioannidis, J. P. A., Mokrysz, C., Nosek, B. A., Flint, J., Robinson, E. S. J., \& Munafò, M. R. (2013). Power failure: why small sample size undermines the reliability of neuroscience. Nature Reviews Neuroscience, 14(May). doi:10.1038/nrn3475

Child, C. (1892). Statistics of "unconscious cerebration". The American Journal of Psychology, 5, 249259. Retrieved from http://www.jstor.org/stable/10.2307/1410868

Cumming, G. (2012). Understanding the new statistics: Effect sizes, confidence intervals, and metaanalysis. NY: Routledge.

Dijksterhuis, A. (2004). Think different: The merits of unconscious thought in preference development and decision making. Journal of Personality and Social Psychology, 87, 586-98. doi:10.1037/0022-3514.87.5.586

Dijksterhuis, A., Bos, M. W., Nordgren, L. F., \& van Baaren, R. B. (2006a). On making the right choice: the deliberation-without-attention effect. Science, 311(5763), 1005-7.

doi:10.1126/science. 1121629 
Dijksterhuis, A., Bos, M. W., Nordgren, L. F., \& van Baaren, R. B. (2006b). Supporting online material for: On making the right choice: the deliberation-without-attention effect. Science, 311(5763), 1005-7. doi:10.1126/science. 1121629

Dijksterhuis, A., \& Nordgren, L. F. (2006). A theory of unconscious thought. Perspectives on Psychological Science, 1, 95-109. doi:10.1111/j.1745-6916.2006.00007.x

Doyen, S., Klein, O., Pichon, C.-L., \& Cleeremans, A. (2012). Behavioral priming: It's all in the mind, but whose mind? PLoS ONE, 7(1), e29081. doi:10.1371/journal.pone.0029081

Elms, A. (1975). The crisis of confidence in social psychology. American Psychologist, (October), 967-976. Retrieved from http://psycnet.apa.org/psycinfo/1976-04477-001

Fanelli, D. (2010). "Positive" results increase down the Hierarchy of the Sciences. PLoS ONE, 5(4), e10068. doi:10.1371/journal.pone.0010068

Fiedler, K., Kutzner, F., \& Krueger, J. I. (2012). The long way from $\alpha$-error control to validity proper: Problems with a short-sighted false-positive debate . Perspectives on Psychological Science , 7 , 661-669. doi:10.1177/1745691612462587

Gall, M., \& Mendelsohn, G. A. (1967). Effects of facilitating techniques and subject-experimenter interaction on creative problem solving. Journal of Personality and Social Psychology, 5, 211-6. doi: $10.1037 / \mathrm{h} 0024130$

Heisenberg, W. (1963). Oral history interview of Werner Heisenberg by Thomas Kuhn. Archive for the History of Quantum Physics, deposit at Harvard University. Cambridge, MA.

Hogg, M. A., \& Grieve, P. (1999). Social identity theory and the crisis of confidence in social psychology: A commentary, and some research on uncertainty reduction. Asian Journal of Social Psychology, 2, 79-93. doi:10.1111/1467-839X.00027

Ioannidis, J. P. A. (2012). Why science is not necessarily self-correcting. Perspectives on Psychological Science, 7, 645-654. doi:10.1177/1745691612464056

Ireland, W. W. (1875). Can unconscious cerebration be proved? The British Journal of Psychiatry, 21(95), 366-387. doi:10.1192/bjp.21.95.366

Kaplan, C. A., \& Davidson, J. (1989). Hatching a theory of incubation effects (No. AIP-98). Pittsburgh, PA: Carnegie-Mellon University of Pittsburgh, Artifical Intelligence and Psychology Project. Retrieved from http://oai.dtic.mil/oai/oai?verb=getRecord\&metadataPrefix=html\&identifier=ADA219275

Ladd, G. (1892). Psychology as so-called "natural science". The Philosophical Review, 1(1), 24-53. Retrieved from http://www.jstor.org/stable/10.2307/2175528

Lakatos, I. (1976). Proofs and refutations: the logic of mathematical discovery (p. xii, 174 p.). Cambridge, NY: Cambridge University Press. 
LeBel, E. P., Borsboom, D., Giner-sorolla, R., Hasselman, F., Peters, K. R., Ratliff, K. A., \& Tucker Smith, C. (2013). PsychDisclosure.org: Grassroot Support for Reforming Reporting Standards in Psychology Perspectives on Psychological Science, in press, 1-10.

LeBel, E. P., \& Peters, K. R. (2011). Fearing the future of empirical psychology: Bem's (2011) evidence of psi as a case study of deficiencies in modal research practice. Review of General Psychology, 15(4), 371-379. doi:10.1037/a0025172

Makel, M. C., Plucker, J. A., \& Hegarty, B. (2012). Replications in psychology research: How often do they really occur? Perspectives on Psychological Science, 7, 537-542. doi: $10.1177 / 1745691612460688$

Molenaar, P. C. M. (2008). On the implications of the classical ergodic theorems: Analysis of developmental processes has to focus on intra-individual variation. Developmental Psychobiology, 50, 60-69. doi:10.1002/dev

Open Science Collaboration. (2012). An open, large-scale, collaborative effort to estimate the reproducibility of psychological science. Perspectives on Psychological Science, 7, 657-660. doi: $10.1177 / 1745691612462588$

Pashler, H., \& Wagenmakers, E.-J. (2012). Editors' introduction to the special section on replicability in psychological science: A crisis of confidence? Perspectives on Psychological Science, 7, 528530. doi:10.1177/1745691612465253

Platt, J. (1964). Strong Inference: Certain systematic methods of scientific thinking may produce much more rapid progress than others. Science, 146(3642), 347-353. doi:10.1126/science.146.3642.347

Rietveld, E. (2008). Special Section: The Skillful Body as a Concernful System of Possible Actions: Phenomena and Neurodynamics. Theory \& Psychology, 18(3), 341-363. doi:10.1177/0959354308089789

Simmons, J. P., Nelson, L. D., \& Simonsohn, U. (2011). False-positive psychology: Undisclosed flexibility in data collection and analysis allows presenting anything as significant. Psychological Science, 22, 1359-66. doi:10.1177/0956797611417632

Sio, U. N., \& Ormerod, T. C. (2009). Does incubation enhance problem solving? A meta-analytic review. Psychological Bulletin, 135, 94-120. doi:10.1037/a0014212

Spencer, H. (1855). The principles of psychology. London, UK: Longman, Brown, Green and Longmans. Retrieved from http://oll.libertyfund.org/title/1394

Stephen, D. G., Dixon, J. A., \& Isenhower, R. W. (2009). Dynamics of representational change: Entropy, action and cognition. Journal of Experimental Psychology: Human Perception and Performance, 35, 1811-1832. doi: $10.1037 / \mathrm{a} 0014510$

Stickgold, R., \& Walker, M. (2004). To sleep, perchance to gain creative insight? Trends in Cognitive Sciences, 8, 191-192. doi:10.1016/j.tics.2004.03.003 
Strick, M., Dijksterhuis, A., Bos, M. W., Sjoerdsma, A., van Baaren, R. B., \& Nordgren, L. F. (2011). A meta-analysis on unconscious thought effects. Social Cognition, 29, 738-762. doi:10.1521/soco.2011.29.6.738

Strick, M., Dijksterhuis, A., \& van Baaren, R. B. (2010). Unconscious-thought effects take place offline, not on-line. Psychological Science, 21, 484-488. doi:10.1177/0956797610363555

Strick, M., Dijksterhuis, A., \& van Baaren, R. B. (2012). Erratum. Psychological Science, 23, 109. doi: $10.1177 / 0956797611432836$

van Dongen, J. (2010). Einstein's unification. Cambridge, NY: Cambridge University Press.

Vul, E., \& Pashler, H. (2012). Voodoo and circularity errors. NeuroImage, 62, 945-948. doi:10.1016/j.neuroimage.2012.01.027

Waroquier, L., Marchiori, D., Klein, O., \& Cleeremans, A. (2009). Methodological pitfalls of the Unconscious Thought paradigm. Judgment and Decision Making, 4, 601-610. doi:??

Wicherts, J. M., Borsboom, D., Kats, J., \& Molenaar, D. (2006). The poor availability of psychological research data for reanalysis. The American psychologist, 61, 726-728. doi:10.1037/0003066X.61.7.726

Wicherts, J. M., Kievit, R. A, Bakker, M., \& Borsboom, D. (2012). Letting the daylight in: Reviewing the reviewers and other ways to maximize transparency in science. Frontiers in Computational Neuroscience, 6:20. doi:10.3389/fncom.2012.00020

Wong, P. (1981). Implicit editorial policies and the integrity of psychology as an empirical science. American Psychologist, 36, 690-691. doi:10.1037/0003-066X.36.6.690

Woodworth, R. S., \& Schlosberg, H. (1954). Experimental psychology. NY: Chicago: Holt, Rinehart and Winston. 